\title{
Quantum Consciousness and the Heart Based Resonant Frequencies Theory
}

\author{
Abdullah Abdulrhman Al Abdulgader* \\ Senior Scientist, Congenital Cardiologist and Invasive Electrophysiologist, Saudi Arabia
}

\begin{abstract}
*Corresponding author: Abdullah Abdulrhman Al Abdulgader, Senior scientist Congenital Cardiologist and Invasive Electrophysiologist, Prince Sultan Cardiac Center (Founder\& Chief Physician), Saudi Arabia.
\end{abstract}

Received Date: January 07, 2021

Published Date: January 26, 2021

\begin{abstract}
The most elusive experience of the human being in this huge endless universe is related to "the human consciousness", human awareness of what is surrounding him, perceiving surroundings nature with deep interceptions and incorporating all of this in the context of emotional experience based on memory and self-identity. The author defines consciousness as" state of alertness and being aware, active and vigilant of the self and surroundings with volition, based in memory and personal identity. This state is ineffable and intrinsic and express itself in presence of soul through activation of different sensing and perceptive body organs but may pass through lighter densities and variable dimensions in quantum nature, if soul leaves the body" [1]. The following discussions in this chapter will expand the understanding in those directions. The sophisticated neurobiological dynamics within the cerebral cortex dominated the scientific views on consciousness science in the current era. However, there is compelling scientific and rational evidence to convince scientific communities that the nature of consciousness involves dynamics inside the skull but essentially much beyond it in extreme dimensions between the skull and the sky [1]. Holistic perception and understanding of human consciousness incorporating other scientific disciplines like the central role of human heart contribution to consciousness, quantum physics, as well as astrobiologigcal aspects of consciousness are critically needed for creation of proper perspective of the nature of human consciousness. Groundbreaking developments in the field with new era of scientific evidence of consciousness beyond the skull, started with shaking the believe and refuting that neuronal firing of brain structures is the sole source of subjective consciousness experience. In addition, the principles of classical mechanics stand handicapped in front of the challenges to explain the true mechanisms operating the secrets of consciousness. We introduce a new integral understanding of human consciousness called the Heart Based Resonant Frequencies [HBRF] Theory of consciousness based on biological and astrophysical experimental records integrating the up to date achieved knowledge in Heart Rate Variability (HRV) and its universal correlates in integration with the current quantum consciousness theories.
\end{abstract}

\section{Resonance in Biology and Astrophysics}

Resonance refers to vibration of large amplitude in electrical or mechanical system caused by a relatively small periodic stimulus of the same or nearly the same period as the natural vibration period of the system. The concept of resonance and its implementations in physiological as well as astrophysical rhythms is of critical significance for life on earth and to human consciousness experience. Common resonant frequencies are what allows different parts of the brain to achieve a phase transition in the speed and bandwidth of information flows between the constituent parts. This phase transition allows for richer varieties of consciousness to arise, with the character and content of that consciousness in each moment determined by the particular set of constituent neurons [2]. The coherence and the delicate organization of the universe is long lasting conceptual direction that affects the applied sciences and points to gigantic governor who master all things at all times. Nikola Tesla is one of the great thinkers in human history. We consider him as our father in the field who inherited to us important heritage of wisdom. An example of this wisdom is his statement "If you 
want to find the secrets of the universe, think in terms of energy, frequency and vibration". Resonance is perceived by us and others as a universal phenomenon that can be shared between all living systems including biological systems and astrophysical ecosystems in away where different systems may resonate in proximity ending up with resonating together at the same frequency, to achieve a shared resonance. Reviewing todays literature demonstrate that resonance as a phenomenon was attractive conceptual part of theories on brain dynamics and consciousness. Compelling concept which deserve full contemplation essential to preserve intellectual alignment between ecosystems is the idea that matter owns mind and certain degree of consciousness is conceivably a feature of all micro and marco creatures. At astrophysical level, the miraculous accuracy of orbiting journeys of the 125 billions galaxies and its stars and plants must have superb intelligent conscious system guiding it over the timeline of life history. At biological level the synchrony and marvelous accuracy of dynamics between human body systems in specific and the diversity of biology cannot be believed to happen without higher intelligent consciousness. What is astonishing and diving us deep in the extreme believe of the gigantic power controlling all life and universe is what we document of the delicate resonance between human heart interbeat intervals referred to as Heart Rate Variability (HRV) and the cosmos as we will discuss later in the next sections.

\section{Human Heart: The King of Organs and its Dominance over the brain}

Revolutionary paradigm has evolved in the last 30 years which has its significant impact on consciousness scientific arena .The inability to explain and match consciousness observations and the failure to reproduce the exact consciousness experience incorporating current knowledge in the field implies presence of deficient rings in the long chain that demand more comprehensive perspective. In this regard we established the King of Organs International Congress for Advanced Cardiac Sciences and held five international congresses (2006,2008,20101,2012 and 2019). The King of Organs congresses are an international collaborative efforts between international renewed scientists in cardiac sciences, psychologists, astrophysicist, mathematicians, geologists, space engineers, signal analysis specialists and other related disciplines. It is chaired by us in Prince Sultan Cardiac Center (Alhasa, Saudi Arabia). Our academic partners are the HeartMath Institute and the Global Coherence Initiative (Boulder Creek, CA, USA), American Institute of Stress (NY, USA), the Global Consciousness Project (Institute of Noetic Sciences, USA), and other western and eastern reputable universities and collaborators. Our mission is to decode the great mystery of consciousness away from the traditional neurobiological approach. Our research areas were neuroradiology, solar and geomagnetic fluctuations and how it affects human autonomic nervous system, quantum physics of the human heart and brain and other related subjects. The heart as the dominant energetic organ of the human body and the role of heart rate variability (HRV) and its orchestrating symphony in the human body and the universe were the illuminators and the distinguished new scientific arena of the King of Organs Congresses. Revolutionary paradigm of the human heart has evolved which awaken ancient wisdom believes and perspective of the human heart as king of organs and master of the human consciousness experience. Scientific discussions that has been the main theme of King of Organs International Conferences was the establishment of the magnificent non pumping functions of human heart, neurologically, biochemically, biophysically and most importantly energetically as the dominant magnetic field generator in human body. In specific, major investigative efforts has been devoted to the discovery that the heart detect stimulus before the brain, brain neural events are locked to heartbeats, Heart Beat Evoked Potentials (HBEPs) and its role in interoception and the human heart connections to the cosmic environments and the far galaxies. The amplitude of the cardiac electrical signal is about 60 times greater compared to the brain while the electromagnetic field of the heart is approximately 5000 times stronger than the brain and can be detected six feet away from the body with sensitive magnetometers [1]. There is a direct mathematical relationship between the HRV patterns, and the spectral information encoded in the magnetic field [3].

\section{The vagal nerve is never vagal: The Afferent Cardiac Neuronal Traffic and Consciousness}

The field of neuroradiology is relatively new discipline which was discussed first time in a scientific conference in King of Organs 2008, Saudi Arabia. The meticulous and sophisticated neurological afferent pathways (Figure 1) as well as energetic dominance of the heart over the brain was astonishing for the modern scientific communities.

Karl Pribram's proposed that low frequency oscillations generated by the heart and body in the form of afferent neural hormonal and electrical patterns are the carriers of emotional information and the higher frequency oscillations found in the EEG reflect the consciousness. John and Beatrice Lacey during 1960s and 1970s created a massive drift in the modern psychophysiological arena. Heart- Brain communication science was revolutionized with their publication declaring that afferent input from the heart and cardiovascular system could significantly affect perception, cognitive functions and behavior which all reflect in conscious experience. The heart behaves as if it had a mind of its own. Laceys showed that patterns of physiological responses were affected as much by the context of a specific task and its requirements as by emotional stimuli, in clear contradiction to Cannon theory of homeostasis which claim constant internal environment, he called the milieu intérieur. Directional fractionation is a phenomenon described by 
Laceys which denotes paradoxical heart rate response as it decelerated and blood pressure decreased, while simultaneously recorded parameters such as, respiratory rate, pupillary dilation and skin conductance all increased as expected. Later, Velden and Wolk demonstrated cognitive performance fluctuated at a rhythm around $0.1 \mathrm{~Hz}$ and showed that the modulation of cortical function was via the heart's influence due to afferent inputs on the neurons in the thalamus, which globally synchronizes cortical activity and the consciousness phenomena $[1,4,5]$. A critical observation here is the finding that "pattern and stability" (of the rhythm) of the heart's afferent inputs, rather than the number of neural bursts within the cardiac cycle that will modulate thalamic activity, which in turn has global effects on brain function and ultimately the consciousness experience $[1,6]$. Growing body of respectful research has since been accumulating indicating that afferent information processed by the intrinsic cardiac nervous system can influence activity in the frontocortical areas and motor cortex, affecting psychological factors and the consciousness experience elements such as attention level, motivation, perceptual sensitivity, and emotional processing [1,6-8]. Astonishing fact of the vagus nerve (means the nerve with unknown role) called sometimes, the tenth cranial nerve is the fact that it has very significant afferent neurons beside its efferent neurons [1]. Around $85-90 \%$ of the fibers in the vagus nerve are afferent [9]. The majority of higher brain centers, as well as emotional experience and cognitive processes are operated by Cardiovascular related afferent neural traffic [10]. The vagus nerve carries afferent connections to the deep nuclei of the brain via the nucleus solitaries (Figure 1). Numerous brain centers including the thalamus, hypothalamus, and amygdala are connected to cardiovascular afferents. Range of frequencies of complex afferent information related to mechanical and chemical factors is continuously sent to the brain and is over time scales ranging from milliseconds to minutes [11]. Vagal afferent nerve stimulation was shown to reduces migraine and cluster headaches and to improve cognitive processing and memory [12]. These afferent connections have multiple consciousness related targets, which include the thalamus, amygdala, reticular formation, hippocampus, raphe nucleus, and the locus coeruleus. Vagus Nerve Stimulation (VNS) will create improved global neurostimulation state leading to promoted spread of cortical signals and caused an increase of metabolic activity leading to behavioral improvement as measured with the Coma Recovery Scale-Revised (CRS-R) scale $[1,13]$.

\section{The Delicate Orchestration of Cardiovascular} System with the Universal Resonances; the Solar and Planetary Geomagnetic Activity

All biological systems on the planet are exposed to an external and internal environment of fluctuating invisible wide range of magnetic fields frequencies. These fields can affect virtually every cell and circuit to a greater or lesser degree. Numerous physiological rhythms have been shown to be synchronized with solar and geomagnetic activity. Geomagnetic and solar influences affect a wide range of human rhythmic systems with the nervous and cardiovascular systems, with their significant contribution to consciousness, being the most clearly impacted [14]. Sharp variations of sudden and sharp nature of geomagnetic, solar activity and its resultant geomagnetic storms can act as stressors, which has the capacity to alter body regulatory processes and rhythmic systems such as melatonin/serotonin balance, blood pressure, breathing, reproductive, immune, neurological, and cardiac system processes [15-18]. In the clinical arena significant increases in hospital admissions for depression, mental disorders psychiatric admission, homicides, suicide attempts, and traffic accidents are associated with planetary geomagnetic disturbances [19-25]. Increase incidence of myocardial infarctions, vascular variability disorders, local and global communication between humans during geomagnetic disturbances are all denotes that brain and cardiovascular systems are clear targets for the planetary geomagnetic disturbances [26-31]. Exacerbation of present disease like development of cardiac arrhythmias and epilepsy is well known during disturbed geomagnetic activity. Clinical evaluation of humans in this prosperous era of geomagnetic knowledge should be done counting at all times the fact that every cell in our body is bathed in an external and internal environment of fluctuating invisible magnetic forces that can affect virtually every cell and circuit in biological systems. Therefore, it should not be surprising that numerous physiological rhythms in humans and global collective behaviors are not only synchronized with solar and geomagnetic activity, but disruptions in these fields can create adverse effects on human health and behavior. The most likely mechanism for explaining how solar and geomagnetic influences affect human health and behavior are a coupling between the human nervous system and resonating geomagnetic frequencies called Schumann resonances, Alfven waves, and other very low frequency resonances that occur in the earth-ionosphere resonant cavity. It is well established that Shumann Resonant frequencies directly overlap with those of the human brain, and the cardiovascular and autonomic nervous systems. Low frequency magnetic oscillations, around $3 \mathrm{~Hz}$, was observed to cause altered EEG rhythms with sedative effect [32]. Applying the lowest Schumann Resonance(SR) frequency of $7.8 \mathrm{~Hz}$ with 90 nano Tesla for 1.5 hours was found to be cardioprotective from stress conditions with reduction of the amount of $\mathrm{CK}$ released to the buffer, during normal conditions, hypoxic conditions and oxidative stress induced by $80 \mu \mathrm{M} \mathrm{H} 202$ [33]. Schumann resonance frequency is 7.83 hertz (Hz), with a (day/night) variation of around $\pm 0.5 \mathrm{~Hz}$. The higher frequencies are $\sim 14,20,26,33,39$ and $45 \mathrm{~Hz}$, all of which closely overlay with alpha $(8-12 \mathrm{~Hz})$, beta $(12-30 \mathrm{~Hz})$ and gamma (30-100 Hz) brain waves. The delicate orchestration of this universal symphony and vibrations with the human autonomic nervous system (ANS) that interacts with cerebral cortex and 
control heart rhythm, respiration, digestive functions and other involuntary activities was investigated. The longest record in human history of human heart rate variability (HRV) synchronized with Solar Wind Indices, Shumann Resonances(SR) and Galactic Cosmic Rays(GCR) monitoring was achieved by our group [34]. We completed long-term study where we examined relationships between solar and magnetic factors and the time course and lags of autonomic nervous system (ANS) responses to changes in solar and geomagnetic activity. Heart rate variability (HRV) was recorded for 72 consecutive hours each week over a five-month period in 16 participants in order to examine ANS responses during normal background environmental periods. Environmental data activity across the study period are seen in Figure 2.

We were able to confirm that changes in solar and geomagnetic activity during periods of normal undisturbed activity affect daily ANS activity. ANS dynamics is known to be critical for human's psychophysiological wellbeing [6]. In another publication, we were able to document significant correlations between the group's HRV and solar wind speed, $\mathrm{Kp}, \mathrm{Ap}$, solar radio flux, cosmic ray counts, Schumann resonance power, and the total variations in the magnetic field [27]. This affect is initiated at different times after the changes in the various environmental factors and persist over varying time periods. Peaks of increased solar activity occurs every 10.5 to 11 years. During those peaks, the sun emits increased ultraviolet (UV) energy and solar radio flux, which is measured by the $2.8 \mathrm{GHz}$ signal (F10.7) [27].

We considered Solar wind intensity as biological stressor as increase in its intensity is well correlated to increase heart rate. Galactic Cosmic Rays (GCR) are highly energetic particles that originate outside the solar system and are likely formed by nuclear explosive events in supernova and other mega giant galaxies. These highly energetic particles consist of fully ionized nuclei ranging from hydrogen, accounting for approximately $89 \%$ of the GCR spectrum, to trace amounts of uranium. The planetary magnetic field and the solar winds are protective for life on earth from this extremely ionized ray. The synchrony of those three critical environmental factors to the human information carrier represented by HRV is shown in Figure 3.

We documented that human HRV with its modulatory effect on the consciousness pillars through ascending neuronal input to cortical and sub cortical structures increases with rise of the three major universal vibrations that we examined: Solar Winds, Shumann Resonances(SR), and the Galactic Cosmic Rays(GCR). This complex interaction between HRV and those environmental energetic fields may contribute to the human knowdelege about the pathomechanistic effects on human psychphysiological homeostasis and the consciousness experience (Figure 3).

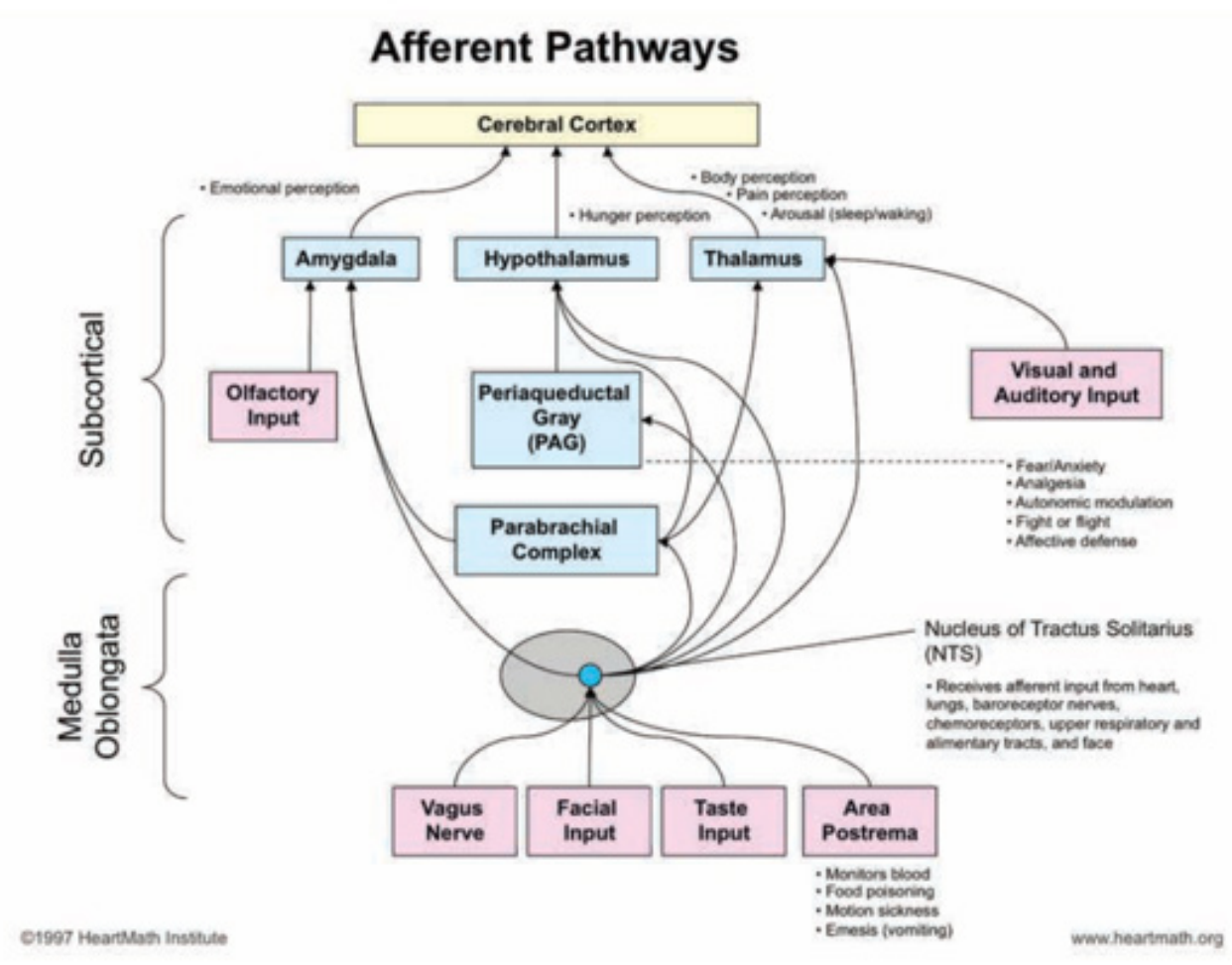

Figure 1: The currently known afferent pathways by which information from the heart and cardiovascular system modulates brain activity. The nucleus of tractus solitarius (NTS) direct connection to the amygdala, hypothalamus and thalamus is shown. 


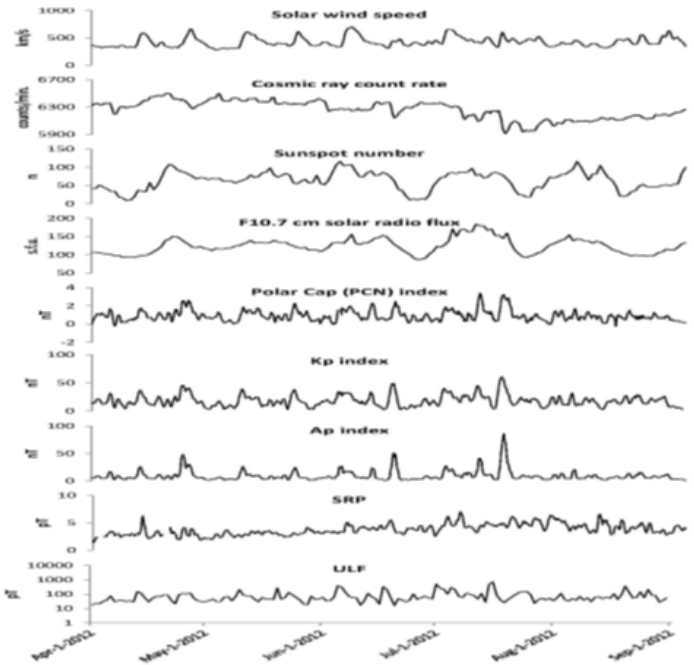

Figure 2: Environmental data activity across the study period. There was a large increase in the Kp and Ap indexes that occurred on July 14th, which resulted from a coronal mass ejection that hit the earth's magnetic field at approximately 1800 UT that day.

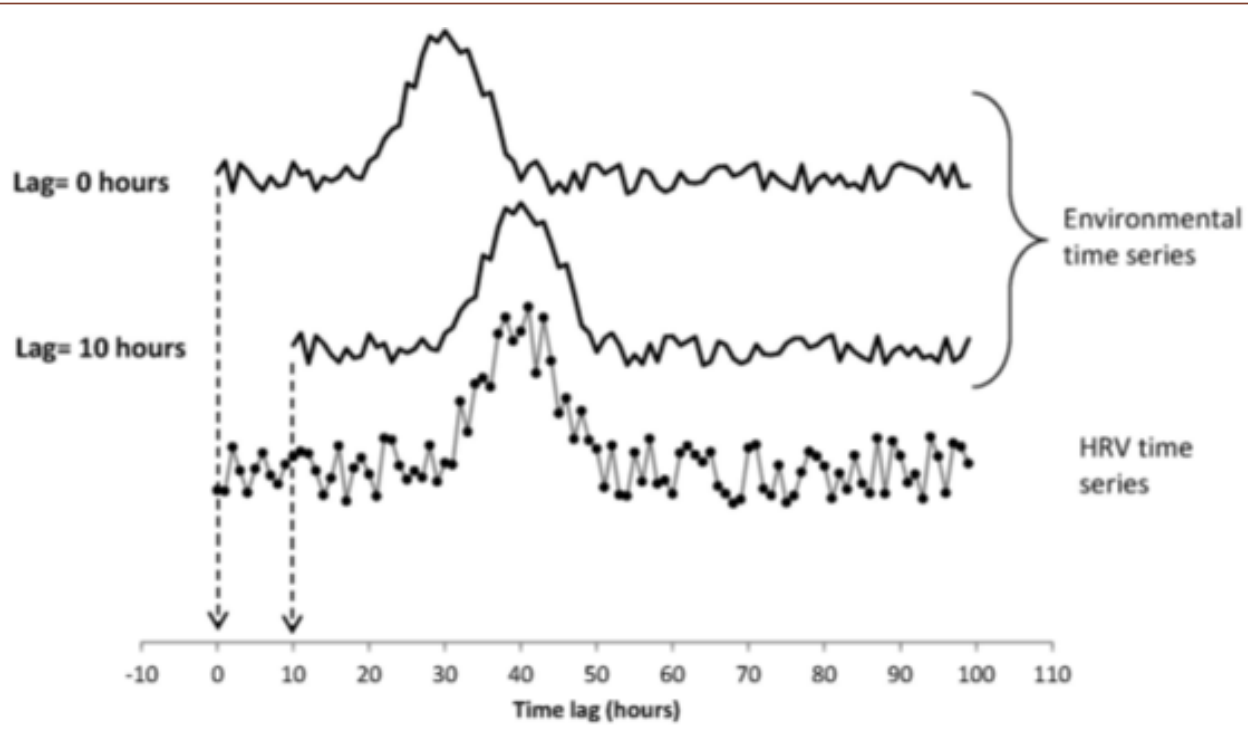

Figure 3: Two data series representing an environmental time series and a HRV time series. Delayed physiological response to the external environmental signal is evident in the upper curve and corrected with 10 hours lag in the second curve.

\section{The Quantum Consciousness}

The idea that quantum mechanics has something to do with the workings of the mind was developed by Eugene Wigner, HungarianAmerican theoretical physicist and Nobel Prize Laurete in Physics in 1963, who proposed that the wave function collapses due to its interaction with consciousness. Freeman Dyson argued that "mind, as manifested by the capacity to make choices, is to some extent inherent in every electron. David Bohm is theoretical physicists who contributed significantly to quantum theory, neuropsychology and the philosophy of mind. He stimulated new era of conceptual approach to consciousness with more fundamental level in the universe. He claimed both quantum theory and relativity pointed to this deeper theory, which he formulated as a quantum field theory. Bohm's proposed implicate order which applies both to matter and consciousness. He suggested that it could explain the relationship between them. Bohm's views mind and matter as projections into our explicate order from the underlying implicate order This more fundamental level was proposed to represent an undivided wholeness and an implicate order, from which arises the explicate order of the universe as we experience it. Holonomic brain theory is a branch of neuroscience investigating the idea that human consciousness is formed by quantum effects in or between brain cells. This specific theory of quantum consciousness was developed by neuroscientist Karl Pribram initially in collaboration 
with David Bohm. In addition to the neuroanatomical components of the human brain including the large fiber tracts in the brain, neurotransmissions also occurs in dendrites and other webs of fine fiber branche, that form webs. Due to the billions of action potentials and neural impulse formations, dynamic electrical fields will result around these dendritic trees. Those dendritic trees can affect other surrounding neurons without physical contact between them by entanglement. In this way, processing in the brain can occur in a non-localized manner. An energy-based concept of information was described by Dennis Gabor, who invented the hologram in 1947, which he described as quanta of information. Later on, he won Nobel prize in physics for this invention in 1971.Karl $\mathrm{H}$ Pribram's holonomic model of brain processing was described in his 1991 Brain and Perception book which include his perspectives on human consciousness with David Bohm. It describes human cognition by modeling the brain as a holographic storage network. Pribram suggests these processes involve electric oscillations in the brain's dendritic networks, which are different from the more commonly known action potentials involving axons and synapses. These oscillations are waves and create wave interference patterns in which memory is encoded naturally. The waves are found to be analyzable by Fourier transform. Gabor, Pribram and others noted the similarities between these brain processes and the storage of information in a hologram. Pribram's holonomic model contributes significantly to human consciousness understanding, specially to the fast-associative memory and the non-locality of memory. In 1991 Orchestrated Objective Reduction (Orch-OR) theory was introduced by physicist Roger Penrose and anesthesiologists Stuart Hameroff. It is a biological philosophy of mind that postulates that at the quantum level consciousness originates inside neurons, rather than the traditional perspective that it is a product of connections between neurons. The interpreting mechanism is contributed to non-computational quantum process performed by quantum bits (qubits) formed collectively on cellular microtubules- called objective reduction. The qubits are based on oscillating dipoles (either electric or magnetic) forming superposed resonance rings in helical pathways throughout lattices of microtubules. Orchestration refers to the hypothetical process by which microtubule-associated proteins (MAPs) and other connective proteins, orchestrate qubit state reduction through modification of space time-separation of their superimposed states. Penrose was faced with a wave of criticism which is in our view not justified as his opponents were too limited with their perspective for human consciousness within the today computational intelligence language. In addition, postulating intuitive thoughts in the context of acceptable scientific language is well known and accepted approach in the philosophy of science. Other important contributors in the field are Hiroomi Umezawa and collaborators who proposed a quantum field theory of memory storage which is fundamentally different from the Penrose-Hameroff theory. In 1967, Hiroomi Umezawa together with L.M. Ricciardi, proposed a quantum theory of the brain which posits a spatially distributed charge formation exhibiting spontaneous breakdowns at micro levels as the basis for processing at macro levels. According to this model, the information resides in the virtual field associated with the dynamics of the cellular matter. Hiroomi Umezawa was known by his extreme originality. His approach was built upon by Karl Pribram and many others and expanded by Giuseppe Vitiello to a dissipative quantum model of brain. Another pioneer in the field is Henry Pierce Stapp. He is American mathematical physicist, known for his work in quantum mechanics who favors the idea that quantum wave functions collapse only when they interact with consciousness. According to Stapp hypothesis alternative quantum possibilities when exposed to conscious mind will select one. His explanation hypothesis differs from that of Penrose and Hameroff. Stapp postulates a process of global collapse through an effect on the synapses by exploitation certain aspects of quantum Zeno effect. David Pearce, British philosopher has conjectured that unitary conscious minds are physical states of quantum coherence (neuronal superpositions). It is clear at this point that scientist from different disciplines over the last 8 decades were trying to come closer to the absolute fact of consciousness. Each theory discussed has its strength and weaknesses, but all lack the comprehensive universal perspective incorporating the origin of consciousness with more homogenous incorporation of current theories in a stronger model capable of bringing us closer to consciousness realm closer than any time ever.

\section{Alabdulgader Theory of Consciousness: The Heart Based Resonant Fields [HBRF]}

\section{Why Heart Based Resonant Frequencies [HBRF] theory}

TheHeartBased ResonantFields [HBRF] theory of Consciousness is based on human heart as the center of human consciousness experience that orchestrate with the planetary and cosmic energetic fields. Comprehensive review of the consciousness science timeline revealed sincere efforts has been spent since early in the twentieth century as witnessed by the elite names in their fields. The twentieth century consciousness theories are distinguished from the old perspective on consciousness nature by the ting and flavor of quantum physics as shown in the previous section. Quantum physics became a true nucleus for other scientific disciplines like neuroscience, psychology, behavioral medicine and others. In spite of all reviewed theories, we believe that scientific communities in the field are still lacking new model of theory with comprehensive integral perspective comprises the major pillars of consciousness from the emanation of consciousness to its conduction and propagation reaching to human energetic fields and frequencies dynamic interactions ending with the fate of conscious experience production and decision making. The failure of the neuronal firing theory which was established by Wilder Penfield's (1891-1976) who adopt the concept that electrical stimulations in certain brain 
areas produce experiential phenomena is one of the major reasons behind our exploration for convincing theory of consciousness [35]. Holistic perspective with spectrum of hierarchical descendant behavior comprising galaxies to genes is immediately needed. This type of perspective demands massive universal indicator capable of encompassing universal frequencies which must be truly representing the universe as astrobiology in one end and humans as biology in the other end. The miraculous choice in this direction is the information and frequencies embedded between human heart beats, known as Heart Rate Variability (HRV). HRV science represent the neurobiology of psychophysiological wellbeing and in the same time the only well documented biological window to the planetary resonance [6]. It is rapidly maturing paradigm that integrates health and wellness observations across a wide variety of biomedical and psychosocial phenomena and illustrates this nonlinear path of development [36]. The transformation of the mathematical calculations of HRV frequencies and its modulation to treat human disease non pharmaceutically, is an emerging and highly promising success [37]. This type of innovative therapeutic models raise our confidence of the inherent capability of the universally correlated frequencies emanating from our bodies to treat human diseases. On the other hand, the delicate orchestration of the human consciousness from beyond the brain became increasingly accepted scientific discussion. Resonances of HRV frequencies with cosmic energetic fields and frequencies is a strategic turning point and new understanding in the history of science. Advances especially, our work and others, in the science of Heart Rate Variability[HRV] and the role of ascending neuronal afferents from the heart to the brain in addition to the miraculous universal symphony between human heart and the universe constitute the most important revolutions in the new understanding [26-34]. Adopting the quantum consciousness theories in the new understanding based on human heart energetic interaction with multiple energetic fields and frequencies constitute the bases of our new understanding of consciousness experience. At this level, it is conspicuous that an integral comprehensive biological and astrophysical evidence of human consciousness based on long term repetitive monitoring of biological indicators synchronized with astrophysical frequencies is highly needed.

\section{Heart based resonant frequencies theory of human consciousness}

Consciousness theories based in neurophilosophy and personal background are accepted scientific trend in the different medical schools including our current western era medicine. Experimental work in the field is astonishingly limited. The perfect direction in the field of consciousness science which was thought as far unrealistic dream is to do a universal based experiment with four cardinal components: First is to choose a measurable indicator of common biological and astrophysical sensitivity which is malleable for Fourier analysis. Second: this indicator must be of true universal expansion to perceive the true consciousness eternal nature. Third is the ability to record the dynamics and responses of this indicator and its synchronicity with ecosystem variables and indicators for as long time period as possible. Fourth is the ability of this indicator to merge easily and in complementary manner with the quantum physics theories if applicable. This seemingly magic indicator was found to be the human Heart Rate Variability(HRV) which was investigated by our Task force Group in the King of Organs conferences (2006,2008,2010,2012,2019), and found to be truly representing the whole mark of human being consciousness experience from the two ends of the massive universal spectrum :the neurobiology of psychophysiological wellbeing at one end and in the other end the planetary resonances [3-7,14]. Based on those solid scientific convections we were able to achieve the longest record of human Heart Rate Variability [HRV][96000 hours] synchronized with the near planetary frequencies, namely the Shumann resonances [SR] and the far, namely Solar Wind indices and the extremely far Cosmic frequencies originating from the endless universal nuclear explosions, namely the Galactic Cosmic Rays[GCR] [38]. Increase in cosmic rays, solar radio flux, and Schumann resonance power was all associated with increased HRV and parasympathetic activity. Complementary to our hypothesis is the heart magnetic field interactions with the brain neuronal electrical fields as described by Karl Pribram as well as the quantum bit state reduction on micro tubules proposed by Penrose and Hamerof and Hiroomi Umezawa and collaborators who proposed a quantum field theory of memory storage. Our hypothesis is based on postulating that there is gigantic source of information contains the most delicate details of the emergence and the fate of all life events and all galaxies and creatures within it. The information is documented and preserved with infinitive accuracy in the form of endless gigantic nuclear energetic fields that we call the Preserved Board[PB].The eternal and the eterral governer of the PB is the miraculous intelligent consciousness of the whole polyverse and all life and beyond. In our domain we call him ALLAH [39]. Our hypothesis of the global consciousness including human consciousness is based on three levels of energetic fields. First is the source of the energy, namely the $\mathrm{PB}$, second is the media of information transmission, namely the massive electrosoliton and the potential magnetic patterns and fields transmitted through the galaxies until earth, third is the receiver, the discriminator, and the magnetic patterns generator namely the heart and its pulsating magnetic field and associates in addition to the entangled electromagnetic fields, neurobiological processes of the brain and electrodermal response. Electrosolitons, which are the charge, energy, and information carriers in the metabolic processes in biological systems, according to Davydov's hypothesis [39], are formed in alpha-helical proteins, DNA, and hydrogen-bonded water molecule chains, present in living cells, intercellular tissues, and in 
all ecological systems [40]. The electromagnetic fields of the universal systems and its associated potential patterns is the information carrier. The dynamic mass of the electrosoliton depends on the frequency of the magnetic field which affect charge transport processes. At the resonant frequency, electrosoliton wave will be drifted by the magnetic field and accordingly emit radiation and sound waves intensively then lose their energy. Electrosolitons emit their own electromagnetic radiation with the frequency of the main harmonic which is dependent on their own velocity. Electromagnetic potential can cause the emergence of the coherent structures that in view of their coherence, openness, and nonlinearity, are able to self-organize and form various hierarchical levels of ecosystems [41]. The human biological system has the ability to induce an electromagnetic field, either through solitons, or during melanin synthesis, which is able to convert a photon into a phonon and vice versa. This electromagnetic resource provides the mechanism for the non-locality, complexity, and self-consistency (self-maintaining) of biological organisms and ecosystems [42]. Via this electrosoliton emitted electromagnetic radiation, subunits of the complex system [cells, organs, individuals, ecosystems] exchange their information which leads to the appearance of the long-range order in a process of the massive hierarchy of ecosystems that is ongoing in descending manner from the galaxies to the local electromagnetic field [EMF] of the heart and brain with impressive accuracy. The HRV of human heart, which is an accurate indicator of the human psychophysiological and consciousness state, is in delicate symphony with the higher energetic fields $[6,38]$. There is a direct mathematical relationship between the HRV patterns which contains our magnetic potentials of our emotions and the spectral information encoded in the magnetic field [7]. This ultimately means that our emotions and consciousness, through our heart energies and fields are truly pulsating and resonate with the far universe in highly organized hierarchical manner. Our new perspective of human consciousness can blend easily in complementary manner with David Bohm's model of the implicate order and its projection, or extrusion into space-time, and with Karl Pribram holonomic mind/brain theory. When the descendant information from the PB transformed by the magnetic fieldsemitted from the interaction of magnetic fields with the massive electrosoliton wave- it interacts with the human heart EMF and brain neuronal entangled fields and neuronal synapses [39]. Psychokinetic process will take over where several encoded choices within the descendant magnetic potential patterns will interact with heart EMF and brain neuronal entangled fields. According to the wave function collapse, the descendant magnetic potential patterns will be reduces to a single pattern at variable time intervals. Von Neumann-Wigner interpretation mentioned about "consciousness causes collapse" in the context of quantum mechanics as general description of the consciousness experience.
Psychokinetic effect of all humanity on collective global consciousness can be thought of with the size of energetic levels fed by over 7 billion beating human hearts to the space. This would be the source of a radiant magnetic potential's energy feeding into an evolving planetary fields. Our postulated underlying mechanism for this conscious decision making step of the HBRF theory is a dual effect of an interaction of the descendant magnetic potential and the neuronal entanglement fields as described by Karl Pribram and the global process collapse through an effect on the synapses with means of quantum Zeno effect as described by Henry Pierce Stapp. Freeman Dyson argument that mind as manifested by the capacity to make choices, is inherent in every electron is in a strong concordance to the wave collapse part of our HBRF theory. We postulate this wave function collapse is the last step occurs between the cerebral neuronal entanglement fields and neuronal synapses interacting with the descendant magnetic potentials before final decision is taken. The heart afferent neuronal inputs to cortical and sub cortical systems and the associate neurotransmitter pathways, are a major player in this process of decision making of one choice over the other. This higher level of orchestrated consciousness from the PB to the neurobiology of heart and brain with the constellation of Information carriers, Electromagnetic force and quantum fields is confined exclusively to humankind for which they are committed to the higher power being responsible about their choices in life. The ancient wisdom with the rooted believe that the human heart is the cradle of the soul and the emanatory of human consciousness as well as the path to ALLAH is, for the first time, in historical intersection with the modern applied sciences [43].

\section{Conclusion}

Human consciousness has been considered to be one of the most elusive issues in life. The time is due to incorporate the huge scientific advances in biology, quantum physics, astrobiology, and philosophy to decode the mystery of consciousness, its nature, propagation and dynamic interactions with biology in general and human consciousness in specific. The concept of resonance and its implementations in our scientific understanding of physiological as well as astrophysical rhythms is of paramount role for what we are calling for in the direction of holistic approach for human consciousness. The failure to reproduce conscious experience with the current mainstream knowledge with neuronal firing of certain brain nuclei and pathways raise the flag to revolutionize our conceptual understanding of consciousness. Ground breaking developments in the direction towards coming closer to the absolute facts is the scientific evidence of the dominance of the human heart as king of organs in the energetic arena and the marvelous developments in the understanding of Heart Rate Variability(HRV) and the associated magnetic field patterns as information carriers. The vagal nerve is not vagal but is a true master of the afferent 
cardiac input to important consciousness related regions in the brain. Our team achievement with the longest record in human history for the HRV as information carrier and its resonances with various cosmic frequencies including Schumann Resonances, Solar Winds indices and Galactic Cosmic Rays (GCR) created massive drift in our basic understanding of consciousness theories. The Heart Based Resonant Fields [HBRF]theory of Consciousness is based on human heart as the center of human consciousness experience that orchestrate with the planetary and cosmic energetic fields. Important quantum consciousness theories are complementary to our HBRF theory. With this level of understanding we are coming closer to decode important aspects of the mystery of human consciousness towards innovations in diagnostic and therapeutic modalities for humanity psychophysiological wellbeing.

\section{Acknowledgement}

None.

\section{Conflict of Interest}

No conflict of interest.

\section{References}

1. Abdullah Abdulrhman Al Abdulgader (2020) Human Consciousness: The role of Cerebral and cerebellar Cortex, vagal afferents, and Beyond, Cerebral and Cerebellar Cortex - Interaction and Dynamics in Health and Disease book, Prof. Stavros Baloyannis (Ed.), Publisher: IntechOpen.

2. Hunt T, Schooler JW (2019) The Easy Part of the Hard Problem: A Resonance Theory of Consciousness. Front Hum Neurosci 13: 378.

3. McCraty R, Atkinson M, Tomasino D, Bradley R (2009) The coherent heart: heart brain interactions, psychophysiological coherence, and the emergence of system-wide order. Integr Rev 5(2):10-115

4. Wolk C, Velden M (1989) Revision of the baroreceptor hypothesis on the basis of the new cardiac cycle effect. In Psychobiology: issues and applications. Amsterdam: Elsevier Science Publishers B.V. 371-379.

5. Lane RD, Reiman EM, Ahem GL, Thayer JF (2001) Activity in medial prefrontal cortex correlates with vagal component of heart rate variability during emotion. Brain Cognit 47: 97-100.

6. Abdullah A Alabdulgader (2017) The human heart rate variability: Neurobiology of psychophysiological well being and planetary resonance. Editorial. Gen Int Med Clin Innov 2(2): 2-4.

7. McCraty R, Atkinson M, Tomasino D, Bradley RT (2009) The coherent heart: heartbrain interactions, psychophysiological coherence, and the emergence of system-wide order. Boulder Creek, CA: Institute of Heartmath.

8. Lane RD, Reiman EM, Ahem GL, Thayer JF (2001) Activity in medial prefrontal cortex correlates with vagal component of heart rate variability during emotion. Brain Cognit 47: 97-100

9. Cameron OG (2002) Visceral Sensory Neuroscience:Interception. New York: Oxford University Press

10. McCraty R, Atkinson M, Tomasino D, Bradley RT (2009b) The coherentheart:heart-brain interactions, psychophysiological coherence, and the emergence of system wide order. Integral Rev 5: 10-115.

11. Armour JA, Kember GC (2004) "Cardiac sensory neurons," in Basic and Clinical Neurocardiology, edsJ.A.ArmourandJ.L.Ardell(NewYork:Oxford UniversityPress): 79-117.

12. Hassert DL, MiyashitaT, Williams CL (2004) The effects of peripheral vagal nerve stimulation at memory-modulating intensity on norepinephrine out put in the basolateralamygdala. Behav Neurosci 118: 79-88.

13. Tiller WA, McCraty R, Atkinson M (1996) Cardiac coherence: a new, noninvasive measure of autonomic nervous system order. Altern Ther Health Med 2: 52-65.

14. Rollin McCraty, Fred Shaffer (2015) Heart Rate Variability: New Perspectives on Physiological Mechanisms, Assessment of Selfregulatory Capacity, and Health Risk. Glob Adv Health Med 4: 46-61.

15. Cherry N (2002) Schumann Resonances, a plausible biophysical mechanism for the human health effects of Solar/Geomagnetic Activity. Natural Hazards 26: 279-331.

16. Ghione S, Mazzasalma L, Del Seppia C, Papi F (1998) Do geomagnetic disturbances of solar origin affect arterial blood pressure? Journal of Human Hypertension 12: 749-754.

17. Hamer JR (1965) Biological entrainment of the human brain by low frequency radiation. Northrop Space Labs: 65-199.

18. Chernouss S, Vinogradov A, Vlassova E (2001) Geophysical Hazard for Human Health in the Circumpolar Auroral Belt: Evidence of a Relationship between Heart Rate Variation and Electromagnetic Disturbances. Natural hazards 23: 121-135

19. Gordon C, Berk M (2003) The effect of geomagnetic storms on suicide. South African Psychiat Rev 6: 24-27.

20. Kay RW (1994) Geomagnetic Storms: Association with Incidence of Depression as Measured by Hospital Admission. British Journal of Psychiatry 164: 403-409.

21. Kay RW (2004) Schizophrenia and season of birth: relationship to geomagnetic storms. Schiz Res 66: 7-20.

22. Nikolaev YS, Rudakov YY, Mansurov SM, Mansurova LG (1976) Interplanetary magnetic field sector structure and disturbances of the central nervous system activity. Reprint N 17a, Acad. Sci USSR, IZMIRAN, Moscow, 29.

23. Oraevskii VN, T K Breus, RM Baevskil̆, SI Rapoport, VM Petrov, et al. (1998) Effect of geomagnetic activity on the functional status of the body. Biofizika 43: 819-826.

24. Halberg F, Cornelissen G, Panksepp J, Otsuka K, Johnson D (2005) Chronomics of autism and suicide. Biomed Pharmacother 59(1): S100108.

25. Berk M, Dodd S, Henry M (2006) Do ambient electromagnetic fields affect behaviour? A demonstration of the relationship between geomagnetic storm activity and suicide. Bioelectromagnetics 27: 151155 .

26. Timofejeva I, McCraty R, Atkinson M, Joffe R, Vainoras A, et al. (2017) Identification of a Group's Physiological Synchronization with Earth's Magnetic Field. Int J Environ Res Public Health 14(9): 998

27. McCraty R, Atkinson M, Stolc V, Alabdulgader AA, Vainoras A, et al. (2017) Synchronization of Human Autonomic Nervous System Rhythms with Geomagnetic Activity in Human Subjects. Int J Environ Res Public Health 14(7): 770.

28. Abdullah Alabdulgade, Rollin McCraty, Mike Atkinson, Alfonsas Vainoras, Kristina Berškiene, et al. (2015) Human Heart Rhythm Sensitivity to Earth Local Magnetic Field Fluctuations. Journal of Vibroengineering: 17.

29. Franz Halberg, Germaine Cornélissen, Rollin McCraty, Jerzy Czaplicki, Abdullah A Al-Abdulgader (2011) Time Structures (Chronomes) of the Blood Circulation, Populations' Health, Human Affairs and Space Weather. World Heart Journal.

30. Abdullah A Al Abdulgader, Germaine Cornélissen Guillaume, Franz Halberg (2011) Vascular Variability Disorders in the Middle East:Case Reports. World Heart Journal.

31. Inga Timofejeva, Rollin McCraty, Mike Atkinson, Roza Joffe, Abdullah A Alabdulgader, Alfonsas Vainoras, Mantas Landauskas, , Minvydas RagulskisGlobal Study of Human Heart Rhythm Synchronization with 
the Earth's Time Varying Magnetic Field,Underr review in the BMC Bioinformatics journal.

32. Belov DR, Kanunikov IE, Kiselev BV (1998) Dependence of human EEG synchronization on the geomagnetic activity on the day of experiment. Ross Fiziol. Zh Im I M Sechenova 84: 761-774.

33. Elhalel G, Price C, Fixler D, Shainberg A (2019) Cardioprotection from stress conditions by weak magnetic fields in the Schumann resonance band. Nature Scientific Reports 9(1)

34. Alabdulgader A, McCraty R, Atkinson M, Dobyns Y, Vainoras A, et al. (2018) Long-Term Study of Heart Rate Variability Responses to Changes in the Solar and Geomagnetic Environment [Internet]. Nature News. Nature Publishing Group.

35. Penfield W (1938) The cerebral cortex in man: I. The cerebral cortex and consciousness. Arch Neurol Psychiatry 40(3): 417-442.

36. Robert Drury JP, Ginsberg Stephen W Porges. Julian F (2019) Thayer: Heart Rate Variability, Health and Well-Being: A Systems Perspective. Frontiers in Public Health | www.frontiersin.org. November 2019 | Volume 7 | Article 323

37. Alabdulgader A (2012) Coherence: a novel nonpharmacological modality for lowering blood pressure in hypertensive patients. Glob Adv Health Med 1(2): 54-62.
38. Alabdulgader A, McCraty R, Atkinson M, Dobyns Y, Vainoras A, et al. (2018) Long-Term Study of Heart Rate Variability Responses to Changes in the Solar and Geomagnetic Environment. Nature Scientific Reports $8(1): 2663$.

39. Abdullah A Alabdulgader (2020) Human Consciousness: The Universal Heart Based Resonant Frequencies and the Massive ecosystems Hierarchy. 9(2).

40. Davydov AS (1985) Solitons in Molecular Systems (2nd edn), Reidel: Dordrecht.

41. Brizhik L, Del Giudice E, Jorgensen, Marchettini N, Tiezzi E (2009) The role of electromagnetic potentials in the evolutionary dynamics of ecosystems. Ecol Modell 220(16): 1865-1869.

42. Adamski A (2020) Soliton perception in the human biological system. Adv Tissue Eng Regen Med Open Access 6(1): 9-13.

43. Abdullah Alabdulgader (2020) The Ancient Wisdom at Intersection with Modern Cardiac Sciences, special issue. Submitted to Cardiology and Vascular Research Journal. ISSN 2639-8486 (under publication). 